\title{
NOVOS ARRANJOS EM TEMPOS DE COVID-19: APOIO REMOTO PARA ATENDIMENTO DE CRIANÇAS COM TRANSTORNO DO ESPECTRO AUTISTA
}

\section{NEW ARRANGEMENTS IN TIMES OF COVID-19: REMOTE SUPPORT TO CARE FOR CHILDREN WITH AUTISM SPECTRUM DISORDER}

\author{
NATALIE BRITO ARARIPE1 - ORCID 0000-0002-2644-7236 \\ AÍDA BRITO1 - ORCID 0000-0002-5691-0025 \\ DANILO CARVALHO DE SÁ1 - ORCID 0000-0003-2810-8278 \\ GIOVANNA FONSECA STEFANI RUGUÊ1 - ORCID 0000-0002-8712-7930 \\ HELOÍSA BUENO MACHADO1 - ORCID 0000-0003-1621-7020 \\ JENNIFER ALVARES TRINDADE BAUER1 - ORCID 0000-0001-6444-3001 \\ JOSÉ UMBELINO GONÇALVES NETO1,2 - ORCID 0000-0002-9182-4341 \\ KeLVIS ROdRIGO SAMPAIO DA CRUZ1,3 - ORCID 0000-0002-5910-5427 \\ LUCELMO LACERDA1,4 - ORCID 0000-0002-9049-5443 \\ 1CLÍNICA LUNA ABA \\ 2UNIVERSIDADE DE FORTALEZA (UNIFOR). BRASIL \\ 3CENTRO UNIVERSITÁRIO UNIFACID WYDEN. BRASIL \\ 4UNIVERSIDADE FEDERAL dE SÃo CARLOS. BRASIL
}

\begin{abstract}
RESUMO
Mesmo no contexto de pandemia e restrições de contato pelo distanciamento social, há a necessidade da continuidade das terapias por via remota ou telemedicina, para treinamento e supervisão de pais na implementação de estratégias de ensino e a continuidade de medidas terapêuticas. O objetivo deste trabalho é discutir alternativas para atenuar os efeitos do distanciamento social sobre o tratamento de crianças com Transtorno do Espectro Autista (TEA). Nessa discussão, propomos maneiras de o analista do comportamento auxiliar as famílias por via remota. Especificamente, abordamos recursos e atividades que podem facilitar aos pais e cuidadores a implementação da nova rotina vivenciada no ambiente domiciliar em períodos de quarentena e distanciamento social. São apontados detalhes nessa implementação, como os cuidados com a organização do ambiente de ensino, o manejo dos reforçadores, a eleição da rotina utilizada, a construção dessa rotina, bem como a sua implementação. Sugerimos um roteiro com algumas perguntas de monitoramento da orientação on-line e finalizamos abordando o Behavior Skill Training para o treinamento e o acompanhamento dos pais na implementação dessas estratégias. $\mathrm{O}$ atendimento remoto por meio de orientação parental na intervenção de Análise do Comportamento Aplicada ao autismo não é algo novo, mas absolutamente necessário em tempos de COVID-19.
\end{abstract}

Palavras-chave: pandemia, Transtorno do Espectro Autista, orientação de pais, rotina visual, telemedicina.

\begin{abstract}
Even in the context of a pandemic with contact restrictions due to social distancing, therapies must continue remotely or by telemedicine, focusing on training and supervision of parents towards implementing instructional strategies as well as keeping therapeutic measures. The purpose of the present paper is to discuss alternatives to mitigate the effects of social distancing on the treatment of children with Autistic Spectrum Disorders (ASD), including how the behavior analyst may help families remotely. It specifically addresses resources and activities that may assist parents and caretakers with the implementation of a new routine in the household during this period of quarantine and social distancing. The details of such implementation are presented, such as cautions to be taken while organizing the teaching environment, managing reinforcers, selecting, building, and executing a routine. As a suggestion, a guideline with a few questions for monitoring on-line guidance is presented. To conclude, the paper approaches Behavior Skill Training for training and monitoring parents while implementing such strategies. Remote therapy through parental guidance in Applied Behavioral Analysis interventions is nothing new, but absolutely essential in COVID-19 times.
\end{abstract}

Keywords: pandemic, Autism Spectrum Disorder, parental guidance, visual routine, telemedicine.

\footnotetext{
Agradecemos a todos e todas profissionais de saúde e pesquisadores(as) que vêm trabalhando na linha de frente do combate ao Corona vírus e a todos e todas trabalhadoras de serviços essenciais e de entrega. Este estudo foi desenvolvido com financiamento próprio. Correspondência para: aba.luna.2020@gmail.com
}

DOI: http://dx.doi.org/10.18542/rebac.v15i2.8768 
Segundo o relatório n. 3, de 2 de abril de 2020, do Núcleo de Métodos Analíticos para Vigilância em Epidemiologia do PROCC/Fiocruz e EMAp/FGV (Grupo MAVE), é bastante acentuado o risco de disseminação da COVID-19 para todos os municípios brasileiros, decorrente da presença de transmissão sustentada em sete centros urbanos: Rio de Janeiro, São Paulo, Belo Horizonte, Porto Alegre, Fortaleza, Brasília e Manaus. Segundo o referido relatório, o primeiro caso importado da COVID-19 foi confirmado no Brasil em 26 de fevereiro de 2020, na cidade de São Paulo, após dois meses do alerta emitido pela China. Os epidemiologistas estimam que "todas as capitais estaduais e o Distrito Federal têm grande probabilidade de acumular casos graves no curto prazo, por ter uma vulnerabilidade geográfica alta para importação de casos, e um contingente grande de população idosa. Em sua maioria, apresentam insuficiência na disponibilidade de leitos hospitalares, respiradores." (Grupo MAVE, 2020, p. 2). Portanto, a recomendação dos especialistas para a desaceleração do número de infecções e óbitos foi de implantação de medidas de restrição do fluxo de pessoas entre municípios, bem como redução do contato entre pessoas dentro das cidades, com as medidas de distanciamento social.

Neste contexto, foram suspensos vários tipos de prestações de serviços, dentre eles as aulas nas escolas, bem como o atendimento psicoeducativo presencial a crianças diagnosticadas com Transtorno do Espectro Autista (TEA) e suas famílias, seja em clínicas ou em domicílio. As famílias se viram na situação imperativa de atender às medidas de distanciamento social. Muitos dos familiares passaram a não ir ao trabalho ou tiveram que fazer home office. As crianças tiveram que se limitar a brincar apenas dentro de casa, evitar contato presencial com amigos e outros familiares, bem como repentinamente se alterou a rotina diária da criança com diagnóstico de TEA de ir à escola, ir à clínica e/ou receber o atendimento domiciliar.

O Transtorno do Espectro Autista é uma condição definida por dois critérios: a) prejuízo na comunicação social, que pode envolver a comunicação verbal, não verbal e a integração entre elas, o reconhecimento e expressão de emoções e a iniciação ou sustentação de interações; b) rigidez comportamental, com interesses fixos, restritos e repetitivos, com estereotipias vocais, motoras e adesão inflexível a rotinas, temas ou objetos (APA, 2014). Estes prejuízos de comunicação podem dificultar a compreensão deste novo contexto social e de novos hábitos de higiene imprescindíveis. A quebra repentina de rotina pode gerar comportamentos desafiadores a estas pessoas, cuja adesão a essas novas rotinas pode se mostrar ritualística.

Que alternativas podemos propor para atenuar os efeitos do distanciamento social sobre o tratamento de crianças com Transtorno do Espectro Autista (TEA)? Como auxiliar as famílias a reorganizarem suas rotinas e as rotinas de seus filhos? Quais atividades de ensino os familiares podem realizar com seus próprios filhos para remediar o período de quarentena?
Nesse contexto de pandemia, sem precedentes para a nossa geração, há a necessidade da continuidade das terapias por via remota ou telemedicina (telehealth), para treinamento e supervisão de pais na implementação de estratégias de ensino e a continuidade de medidas terapêuticas. Felizmente, pesquisas nessa área têm crescido nos últimos anos, demonstrando ser possível o tratamento (Blanco, Meisels, Blair, \& Leonard, 2020; Ferguson, Craig, \& Dounavi, 2019; Peterson, Piazza, Luczynski, \& Fisher, 2017; Silva, Barboza, Miguel, \& Barros, 2019; Vismara, McCormick, Young, Nadhan, \& Monlux, 2013). Blanco e colaboradores (2020) destacam alguns passos para a implementação do serviço de telemedicina em análise do comportamento aplicada ao TEA, dentre eles considerar os suportes visuais que serão usados e identificar os reforçadores e como serão dispensados.

No presente artigo, serão discutidas algumas diretrizes levantadas pela literatura acerca de três tópicos fundamentais para essa modalidade de atendimento: 1 . Organização da rotina visual pelos pais para as tarefas dos seus filhos; 2. Manejo de arranjos ambientais pelos mesmos cuidadores para a otimização dos procedimentos terapêuticos e 3. Acompanhamento remoto desses pais na implementação desses novos arranjos.

\section{ORGANIZANDO A ROTINA VISUAL}

A resistência a transições e modificações em atividades rotineiras ou não planejadas nos indivíduos com TEA está relacionada à ausência de previsibilidade (Flannery \& Horner, 1994). A implementação de rotina visual é uma prática baseada em evidências que possui função de organização ambiental, controle de expectativas em torno de atividades, fornecimento de dicas ou lembretes, assim como fornecimento de instruções (National Autism Center, 2015). É benéfica para o indivíduo com TEA, pois oferece a previsibilidade acerca dos acontecimentos futuros e garante uma maior colaboração e rendimento da criança nas atividades, beneficiando também o controle da sua ansiedade com relação aos acontecimentos (Fialho, 2018).

A quinta edição do Manual Estatístico e Diagnóstico de Transtornos Mentais - DSM-5 descreve dois critérios diagnósticos para o Transtorno do Espectro Autista: o primeiro é o prejuízo na Comunicação Social e o segundo é a presença de padrões restritos e repetitivos de comportamento, interesses ou atividades, que é descrito em uma série de exemplos, e um deles é "adesão inflexível a rotinas" (APA, 2014, p. 50). Isso demonstra que há um consenso científico de que haja uma alta prevalência desta forte adesão a rotinas na população com TEA, necessitando, portanto, que a levemos em consideração ao trabalhar com este público.

Não se sabe exatamente porque pessoas com TEA aderem de maneira inflexível a rotinas, mas Flannery e Horner, em 1994, testaram a hipótese de que isso ocorre devido à previsibilidade que a rotina oferece, isto é, que é mais reforçador, para uma parte dessas crianças, se comportar de uma maneira previsível. Flannery e Horner 
(1994) trabalharam com uma linha de base reversa entre tratamentos alternativos, $\mathrm{ABAB}$, testando (a) tarefas familiares e desconhecidas, (b) tarefas desconhecidas durante condições previsíveis (sinalizadas) e imprevisíveis (não sinalizadas) e (c) tarefas desconhecidas repetidas em várias sessões (por exemplo, dentro e entre fases). Durante a linha de base, problemas de comportamento eram ignorados e redirecionados para a atividade e atividades desconhecidas e conhecidas foram testadas com e sem previsibilidade. A variável dependente era o engajamento em comportamento-problema.

Com esse delineamento, os pesquisadores fizeram diferentes atividades com duas crianças com níveis de moderado a severo de TEA e Deficiência Intelectual. Algumas dessas atividades já eram conhecidas pelas crianças, a que chamaram de "familiares", e algumas ainda eram desconhecidas, a que chamaram de "não familiares", e ambos os tipos foram oferecidos na condição "previsível" e "não previsível". Isto é, antes do começo das atividades, eles apresentavam uma rotina do dia para as crianças, de modo que algumas atividades já eram previsíveis (porque tinham sido apresentadas na rotina) e outras eram imprevisíveis (porque não tinham sido apresentadas na rotina).

Neste estudo de Flannery e Horner (1994), a variável independente que mais reduziu o comportamentoproblema não foi o prévio conhecimento da atividade em questão, mas a previsibilidade da atividade, isto é, mesmo que uma tarefa fosse não familiar, se esta atividade estivesse na rotina da criança, então ela apresentaria menos comportamentos-problema do que mesmo uma atividade já familiar que lhe fosse apresentada abruptamente, sem que estivesse previamente estabelecida na rotina diária da criança.

Nos dois casos analisados pelos autores, a avaliação funcional demonstrou que a função do comportamento-problema nas condições não previsíveis eram de fuga, isto é, eles se comportavam para sair da nova tarefa e retornar à rotina previamente apresentada $\mathrm{e}$, portanto, para ter acesso às tarefas previsíveis, mesmo que fossem não familiares.

Os autores reconhecem a limitação da validade externa do estudo, que é baixa, devido à quantidade de participantes, mas sugerem que em casos semelhantes, em que a análise funcional indicar que os comportamentosproblema gerados em contextos de transição forem controlados por fuga, que se pudesse estabelecer ambientes consistentes e previsíveis, incrementando assim o tempo de engajamento em atividades produtivas destas crianças (Flannery \& Horner, 1994).

Após isto, muita tecnologia de apresentação de rotina de maneira visual e verbal foi desenvolvida e testada no âmbito das intervenções comportamentais ao TEA, formando um conjunto de pesquisas bastante robusto acerca de vários diferentes tipos de rotinas, que apresentam prós e contras dessas rotinas visuais e verbais (Sterling-Turner \& Jordan, 2007). A rotina visual, portanto, vem sendo considerada como uma Prática Baseada em Evidências (National Autism Center, 2015; Wong et al., 2015), sendo aplicada em escolas que trabalham com pessoas com TEA e em intervenções comportamentais, no setting de implementação e também nas casas de pessoas com TEA.

A rotina visual possibilita demonstrar com imagens as alterações dentro de um roteiro previamente apresentado (Cohen \& Sloan, 2007). As modificações na rotina devem ser antecipadas e demonstradas visualmente através do quadro de rotina, com a retirada ou colocação da figura representativa da tarefa e sua substituição por outra, podendo o processo ser realizado pela própria criança com ajuda dos pais. Neste contexto, será a previsibilidade da mudança que diminuirá a possibilidade da ocorrência de comportamentos-problema com a quebra da rotina previamente apresentada (Dettmer, Simpson, Myles, \& Ganz, 2000; Hume \& Smith, 2009; Massey \& Wheeler, 2000; Morrison, Sainato, Benchaaban, \& Endo, 2002; Smith \& Collet-Klingenberg, 2009).

As rotinas visuais são organizadas em agendas/quadros de rotina diários, semanais e até mensais, com as atividades representadas através de figuras ou fotografias. Elas são usadas para ilustrar a atividade que está sendo realizada, as concluídas e as que virão, e esse recurso também possibilita demonstrar visualmente as cotidianas alterações possíveis dentro de uma rotina (Cohen \& Sloan, 2007).

$\mathrm{Na}$ implementação, terapeuta e pais devem programar objetivos semanais e planejar como apresentálos. Como sugestão, as imagens podem ser organizadas da seguinte forma: durante o dia, os pais podem apresentar a rotina seguindo a passagem das tarefas conforme forem ocorrendo e identificando para a criança a tarefa concluída, a tarefa a ser iniciada e a que vem depois; durante a passagem da rotina, os pais nomeiam as tarefas de forma simples e, se necessário, podem auxiliar no direcionamento para a atividade e garantir sua execução, fazendo seu redirecionamento para o painel de rotina ao final da tarefa, e repetir o processo seguindo a passagem das mesmas (Dettmer et al., 2000; Hume \& Smith, 2009).

Fialho (2018) afirma que a criança também pode participar da montagem da rotina diária. Esta pode ser uma oportunidade para ela se comunicar, de modo que os pais podem aproveitar a montagem da rotina visual como um recurso para estimular as verbalizações, incentivando a criança a nomear as tarefas ou fazendo construções ainda mais complexas com elaborações de frases que descrevam a ordem das atividades. É também uma oportunidade para reforçar comportamentos de escolher e tomar decisões, pois se pode dar a oportunidade de a criança sugerir a ordem de suas atividades quando desejar fazer uma atividade antes da outra.

Para que qualquer rotina seja implementada, os horários das atividades a serem realizadas são essenciais para o processo funcionar. Depois dos horários e necessidades de cada criança, as figuras são construídas de acordo com as prioridades dela e do ambiente. Além de figuras, pode-se pensar em dicas visuais escritas, caso sejam crianças alfabetizadas e demonstrem uma resposta melhor a material escrito (Hume \& Smith, 2009).

Essas sugestões de implementação de rotinas visuais podem ser realizadas para diferentes atividades, 
desde atividades de vida diária (por exemplo: vestir-se, escovar os dentes, tomar banho etc.), até atividades específicas dentro de um programa terapêutico (por exemplo: pintar, desenhar, cortar, ler etc.). O tamanho e a especificidade da rotina dependem do repertório do aprendiz e a sua ampliação deve ser feita gradativamente.

As rotinas podem ser feitas para qualquer idade, com o intuito de aumentar o envolvimento da tarefa e interação social. Tais ganhos são evidentes quando os arranjos ambientais são organizados para maximizar os efeitos dos procedimentos implementados através das rotinas e agendas visuais.

\section{MANEJANDO OS ARRANJOS AMBIENTAIS}

Com a impossibilidade de o profissional realizar os procedimentos presencialmente, um dos pontos iniciais e fundamentais da orientação on-line aos cuidadores é o da organização do ambiente e dos materiais para realizarem as atividades com a criança.

Segundo Windholz (2016), o ambiente físico para desenvolver as estimulações precisa apresentar algumas características específicas, afinal, para que o local facilite a condução dessas atividades, sejam elas estruturadas ou não, a organização do ambiente pode atuar favorecendo ou desfavorecendo sua realização e a própria aprendizagem. O objetivo é maximizar as condições ambientais para aumentar a probabilidade de generalização das habilidades que já foram adquiridas em um ambiente estruturado de terapias. Essas habilidades podem englobar diversas formas de comportamentos operantes, como habilidades acadêmicas, motoras, entre outras.

No geral, as terapias ocorrem em ambientes estruturados, com equipamentos necessários e adequados, mas quando pensamos na realidade de uma pandemia, as estimulações podem ser realizadas no dia a dia da criança, utilizando os próprios itens e situações naturais que o ambiente de casa oferece. Um exemplo seria a execução das Atividades de Vida Diária (AVD), que podem ser realizadas tanto em locais estruturados, como em locais de recreação e ao ar livre (Windholz, 2016).

$\mathrm{Na}$ tentativa de facilitar a elaboração de uma rotina e a organização do ambiente, precisamos pensar prioritariamente: a) nas reais condições que os cuidadores possuem, b) nas atividades que consideram prioridades (Sella \& Ribeiro, 2018) e c) nas preferências do indivíduo.

Ao pensarmos nas condições dos cuidadores, levantamos a disponibilidade deles e os horários possíveis para encontro on-line, com objetivo de auxiliarmos nessa organização do ambiente. A partir daí, podemos auxiliálos a montar uma rotina para eles próprios (cuidadores) com horários específicos para a organização do ambiente. Outro ponto relevante aqui é a organização da casa ou do apartamento em microambientes com funções específicas, para aumentar o controle de estímulos sobre determinadas classes de respostas. Por exemplo, conforme as especificidades de cada residência: organizar um cômodo da casa para terapia e atividades da escola, outro cômodo para descanso e outro para atividades de lazer. Tal divisão dos espaços pode ser um sinalizador extra da divisão da rotina.
Sobre as prioridades, como o contexto de ensino em casa em geral é bastante diferente da escola ou clínicas, é importante também repensar os objetivos do Plano de Ensino Individualizado. Num contexto de pandemia e distanciamento social, a prioridade deve ser para a generalização e manutenção das habilidades já aprendidas, levando em consideração o que é possível de ser realizado pela família.

Após identificar os momentos possíveis de realização das atividades, planejar a organização do ambiente e delimitar as prioridades sobre o que intervir, o terapeuta auxilia a família na organização do material. Para facilitar essa organização, os pais devem ser instruídos a, primeiro, estruturar os materiais em categorias e, em seguida, retirar do ambiente de ensino estímulos desnecessários e possíveis distratores, contudo, sem impor ambientes higienizados demais, livres de quaisquer estímulos.

O ambiente de ensino deve exercer um controle de estímulos adequado para evitar distração, contendo somente os materiais necessários, como estímulos a serem apresentados e reforçadores, que devem estar presentes e ao alcance da pessoa que irá realizar o treino (Fialho, 2018). A reorganização dos itens de casa para evitar acidentes domésticos também é uma importante diretriz a ser orientada nesse momento.

A separação dos materiais por categoria proporciona agilidade durante o treino, além de aumentar a familiaridade com os estímulos que serão utilizados. O terapeuta, por já conhecer as habilidades da criança, orienta os pais na organização dos materiais para treino em categorias, como: grupos de jogos, frutas, carros, brinquedos, material acadêmico, itens reforçadores em geral, brinquedos de imaginação, materiais que podem ser utilizados para reforço social, jogos de engajamento, material de encaixe, materiais sensoriais e pareamentos.

Vale destacar que a separação dos estímulos é importante para viabilizar a apresentação do material que seja apropriado para o treino de uma habilidade específica. Por exemplo, o terapeuta pode orientar a família a separar em uma caixa os estímulos similares de higiene (por exemplo: dois pentes, duas escovas de dentes, duas esponjas) para a realização de um treino de pareamento por semelhança. Inúmeros "kits" de estímulos como esse podem ser criados a partir de um planejamento pensado nas habilidades da criança e nos recursos que a família tem em casa.

Além dos pontos levantados para o planejamento de uma rotina e a organização do ambiente domiciliar, para a continuidade das intervenções, os pais ou outros cuidadores primários da criança, precisam lidar com a manipulação de variáveis motivacionais que interferem no processo do ensino/manutenção das habilidades.

Chamamos, na literatura comportamental, essas variáveis motivacionais de operações motivadoras (Michael, 1982; Miguel, 2000). Michael (1982) chama a atenção para a efetividade momentânea de um evento enquanto reforçador, quando privação e saciação do estímulo estariam em operação. Trata-se, entre outras palavras, de uma situação em que o valor de um item 
reforçador modula sua efetividade. Como por exemplo, no caso de privação de tablet (ficar horas sem brincar com o aparelho) tornaria o valor reforçador de "tablet" mais efetivo diante do comportamento exigido, como pedir por tablet.

O profissional supervisionando as famílias neste contexto de distanciamento social, além de orientá-las na organização dos materiais e do ambiente de intervenção em casa, também deve dar orientações sobre a utilização dos itens de preferência do filho, que serão os estímulos potencialmente reforçadores, fundamentais para manter o aprendiz engajado nas atividades propostas no plano terapêutico.

Por exemplo, digamos que ao longo do dia os pais observam que seu filho(a) se aproxima, olha, pega ou pede por um item (um brinquedo, por exemplo). O item, nesse contexto, está funcionando como um estímulo seletor, com função discriminativa (Cumming \& Berryman, 1965; Debert, Matos, \& Andery, 2006), um indicativo de que há uma operação estabelecedora. Nesse sentido, torna os comportamentos de se aproximar, olhar, pegar e pedir mais prováveis, tendo em vista que esses comportamentos foram aprendidos no passado na interação com este item, este exercendo uma função evocativa (Michael, 1982).

Com base nisso, é possível que os pais possam manejar o acesso aos itens durante os treinos no ambiente de casa. Em um estudo aplicado, Hall e Sundberg (1987) desenvolveram um procedimento chamado cadeia interrompida. Um exemplo disso para o atual contexto seria o adulto apresentar um quebra-cabeças de três peças onde ele monta duas peças deixando a última ausente, nesta ocasião o fato de o item faltante estar ausente tem função de operação estabelecedora, pois evoca o comportamento de pedir pela peça.

O profissional deve orientar que os pais façam uma lista com os brinquedos que são de uso frequente do filho (que possivelmente são os com maior valor reforçador) e com os brinquedos que o filho possui mas que não usa com tanta frequência (que possivelmente são de menor valor reforçador). Então, devem separar os materiais do filho por itens ou por áreas (Windholz, 2016) e por tipo de reforçadores, como por exemplo: tangível, comestível, social, sensorial. O profissional pode entregar uma tabela com essas categorias para os pais preencherem, facilitando-lhes a organização dos estímulos reforçadores.

Uma avaliação de itens potencialmente reforçadores pode ser utilizada, como é o caso do teste de preferência por observação de operante livre (Roane, Vollmer, Ringdahl, \& Marcus, 1998). Nesta avaliação, os pais podem escolher dez ou mais brinquedos ou itens usualmente preferidos pelo filho, espalhá-los no chão em um espaço previamente organizado, como, por exemplo, sobre um tapete no espaço onde a criança costuma brincar. Antes de iniciar a avaliação, os pais podem disponibilizar esses brinquedos ou itens, um de cada vez, e permitir que o filho os utilize livremente. Em um outro momento, devese disponibilizar todos os brinquedos diante da criança e dar a orientação para que utilize qualquer um dos brinquedos. Nesse momento, os pais devem anotar quais foram as escolhas por um determinado tempo, por exemplo em 5 minutos de brincadeira.

Por ser menos associada à emissão de comportamentos-problema, esta forma de avaliação de preferências tem sido uma grande indicação para os pais que estão no papel de aplicadores, visto que ela implica em poucas demandas e não há restrição de acesso ou retirada dos itens de preferência (para mais detalhes, conferir Sella \& Ribeiro, 2018, p. 115).

$\mathrm{O}$ sucesso no manejo de consequências reforçadoras depende fundamentalmente de habilidades do aplicador (Durigon \& Matheus, 2018). Assim, as expectativas sobre a intervenção feita pelos pais deve ser um tópico a ser conversado, pois se deve ter em mente que, em geral, é um grande desafio aos pais identificar e manejar consequências reforçadoras para os comportamentos mais relevantes de seu filho, conhecer as propriedades dos diferentes tipos de reforçadores, além de, quando possível, ensinar novos reforçadores (Dozier, Iwata, Thomason-Sassi, Worsdell, \& Wilson, 2012; Longano \& Greer, 2006).

A condução de programas de ensino focados em treinos naturalísticos apresenta uma possibilidade promissora para a intervenção feita pelos pais no contexto de pandemia, tendo em vista que tais procedimentos utilizam como consequência o reforço intrínseco (Comunidad Los Horcones, 1992; Ferster, 1967). Reforçadores intrínsecos são consequências reforçadoras produzidas diretamente pelo comportamento do indivíduo, sendo resultados automáticos e naturais do responder (Comunidad Los Horcones, 1992). Por exemplo, o comportamento de brincar tem como reforçador intrínseco as variadas formas de autoestimulação produzidas na brincadeira e a continuação da atividade em si (Yamamoto \& Carvalho, 2002). Isso difere, por exemplo, do comportamento de fazer uma tarefa escolar que seja recompensada por pontos, que neste caso estaria sendo mantida por reforçamento extrínseco (os pontos). Um reforçamento intrínseco do fazer a tarefa escolar seria, por exemplo, a própria aprendizagem de uma certa habilidade acadêmica.

Assim, uma brincadeira com blocos de encaixar, por exemplo, pode ser uma oportunidade para se realizar um treino de imitação com o objeto, cuja consequência reforçadora intrínseca seria a observação dos objetos se empilhando, formando um objeto maior, caindo no chão e se espalhando, bem como pela interação com pais, cuidadores e/ou irmãos em tom de diversão, obtidos na própria atividade lúdica. Na tentativa de ensino com este brinquedo, a imitação da criança seria reforçada pela continuação no acesso ao brinquedo e na liberdade de brincar como desejar, conforme Schreibman et al. (2015) sugerem.

Um método adicional para aumentar a motivação envolve intercalar atividades de maior domínio pela criança (mais fáceis) com atividades mais difíceis para ela, ou seja, intercalar atividades que demandam habilidades já aprendidas com atividades que demandam habilidades novas ou em processo de aprendizagem (Schreibman et al., 2015). Como por exemplo, intercalar 20 minutos de 
atividade de pareamento com 20 minutos de vídeo do desenho favorito. O objetivo dessa estratégia é aumentar a motivação da criança, diminuir a frustração devido ao fracasso e manter as habilidades já aprendidas.

Como se pode perceber com as diretrizes expostas, o arranjo do ambiente, de operações motivadoras e da rotina visual pressupõem o conhecimento de inúmeras estratégias por parte do terapeuta. A forma como essas estratégias serão repassadas aos cuidadores, bom como seu acompanhamento, serão discutidas na próxima seção.

\section{ACOMPANHAMENTO E MONITORAMENTO REMOTO}

De um modo geral, o novo papel assumido pelos cuidadores em época de pandemia estará sob orientação de um profissional que manterá contato por via on-line, o qual usará a tecnologia para orientar um treino de habilidades comportamentais à distância.

Para isso, profissional e pais devem ficar sob controle final de um monitoramento partilhado, no qual as duas pontas desse processo interventivo - família e terapeuta - possam acompanhar todo o processo descrito acima: avaliar habilidades, conduzir treino de pais, dispensar reforçadores e organizá-los, usar rotinas visuais e modelar respostas na criança.

Esse monitoramento partilhado pode ser efetuado através do próprio acompanhamento de intervenções online (síncrono) ou pode ser feito através de feedback sobre as filmagens produzidas pelos próprios cuidadores (assíncrono), cuja lista de critérios pode abranger a seguinte estrutura:

1. Planejei minha rotina e do meu filho?

2. Usei a rotina visual para isso?

3. Organizei o ambiente e os reforçadores?

4. Trabalhei as habilidades escolhidas para o dia?

5. Fiz os registros para discutir com o terapeuta remotamente?

Essas ideias estão presentes e dispersas já desde o início do crescimento da Análise do Comportamento, como podemos ler em Graziano (1969) e Berkowitz \& Graziano (1972). O compartilhamento de técnicas comportamentais deve ser entendido como procedimentais possíveis para o treinamento de pais, já que os mesmos possuem responsabilidades morais, éticas e legais, bem como o maior grau de contato e controle do ambiente dos seus filhos.

O treinamento de pais pode ser apoiado em um ensino estruturado de habilidades feito pelo terapeuta, o que, na literatura analítico-comportamental, é também denominado de Behavior Skill Training (BST). O BST é um procedimento de ensino feito em 4 etapas: Instrução, modelação, ensaio e feedback (Johnson et al., 2005, 2007). Versões adaptadas desse tipo de treinamento podem ser aplicadas no contexto de pandemia, via protocolos semanais com:

1. Orientações aos pais sobre a habilidade a ser treinada, por meio de vídeos produzidos pelo terapeuta e protocolos de ensino. - instrução e modelação.
2. Observação de vídeos produzidos pelos pais com o próprio filho e acompanhamento das intervenções por eles aplicadas, com feedback de correção ou reforçamento - ensaio e feedback.

A estratégia do BST pode ser uma excelente ferramenta aliada na estruturação e no acompanhamento remoto dos cuidadores, não só em tempos de COVID-19, como também na lide diária do Analista do Comportamento.

\section{CONSIDERAÇÕES FINAIS}

$\mathrm{O}$ atendimento remoto por meio de orientação parental na intervenção de Análise do Comportamento Aplicada ao TEA não é algo novo, mas absolutamente necessário em tempos de COVID-19. Discutimos, nesse artigo, algumas estratégias, embasadas na literatura, para a estruturação e o acompanhamento de uma intervenção analítico comportamental nesses moldes.

Enfatizamos a importância da estruturação da rotina visual e da estruturação do ambiente e fornecemos algumas diretrizes para a organização de ambos. Sugerimos um roteiro com algumas perguntas de monitoramento da orientação on-line e finalizamos abordando o Behavior Skill Training para o treinamento e o acompanhamento dos pais na implementação dessas estratégias.

Frente à urgência imposta pela pandemia de orientações e produções, especialmente em língua portuguesa, para uma intervenção analíticocomportamental remota para pessoas com TEA, produzimos esse artigo com as diretrizes abordadas. Não obstante, ressaltamos a importância de estudos empíricos de longo prazo acerca da efetividade da implementação desses protocolos em contextos de urgência e no contexto brasileiro.

\section{POTENCIAL CONFLITO DE INTERESSES}

Os autores atuam como prestadores(as) de serviço analítico-comportamentais para pessoas diagnosticadas com TEA.

\section{CONTRIBUIÇÃO DE CADA AUTOR}

Certificamos que todos os autores participaram suficientemente do trabalho para tornar pública sua responsabilidade pelo conteúdo. A contribuição de cada autor pode ser atribuída como se segue: Natalie Brito Araripe, Aída Brito e José Umbelino Gonçalves Neto foram responsáveis pela delimitação dos pontos teóricos a serem discutidos, bem como pela organização e revisão teórica e metodológica do trabalho; José Umbelino Gonçalves Neto e Lucelmo Lacerda contribuíram com a introdução e contextualização das discussões; Heloísa Bueno Machado e Natalie Brito contribuíram com os apontamentos sobre a organização do ambiente; Danilo Carvalho de Sá e Kelvis Rodrigo Sampaio da Cruz contribuíram com a discussão sobre o manejo de Operações Motivadoras; Aída Brito, Giovanna Fonseca Stefani Ruguê e Jennifer Alvares Trindade Bauer contribuíram com os apontamentos sobre a organização e implementação de rotinas visuais. 


\section{DIREITOS AUTORAIS}

Este é um artigo aberto e pode ser reproduzido livremente, distribuído, transmitido ou modificado, por qualquer pessoa desde que usado sem fins comerciais. $\mathrm{O}$ trabalho é disponibilizado sob a licença Creative Commons 4.0 BY-NC.

\section{(cc) BY-NO}

\section{REFERÊNCIAS}

American Psychiatric Association (2014). DSM-5: Manual diagnóstico e estatístico de transtornos mentais. São Paulo: Artmed Editora.

Berkowitz, B. P., \& Graziano, A. M. (1972). Training parents as behavior therapists: A review. Behaviour Research and Therapy, 10(4), 297-317. doi: 10.1016/0005-7967(72)90054-X

Blanco, S., Meisels, M., Blair, B. J, Leonard, L. (2020, março). Telehealth: Evidence-based practice in the time of COVID-19. Recuperado de: https://bhcoe.org/2020/03/telehealth-aba-therapy-ebpcovid-19/

Cohen, M. J., \& Sloan, D. L. (2007). Visual supports for people with autism: A guide for parents and professionals. Bethesda: Woodbine House.

Cumming, W. W., \& Berryman, R. (1965). The complex discriminated operant: Studies of matching to sample and related problems. In: D. I. Mostofski (Ed.), Stimulus generalization (pp. 284-329). Stanford: Stanford University Press.

Debert, P., Matos, M. A., \& Andery, M. A. P. A. (2006). Discriminação condicional: Definições, procedimentos e dados recentes. Revista Brasileira de Análise do Comportamento, 2(1), 37-52. doi: 10.18542/rebac.v2i1.801

Dettmer, S., Simpson, R. L., Myles, B. S., \& Ganz, J. B. (2000). The use of visual supports to facilitate transitions of students with autism. Focus on autism and other developmental disabilities, 15(3), 163-169. doi: $10.1177 \% 2 F 108835760001500307$

Dozier C. L., Iwata, B. A., Thomason-Sassi, J., Worsdell, A. S., \& Wilson, D. M. (2012). A comparison of two pairing procedures to establish praise as a reinforcer. Journal of Applied Behavior Analysis, 45(4), 721-735. doi: 10.1901/jaba.2012.45-721

Durigon, L. T., \& Matheus, N. M. (2018). Esquemas de reforçamento e o uso de economia de fichas. In: C. P. Duarte, L. C. Silva, \& R. L. Velloso (Orgs.), Estratégias da análise do comportamento aplicada para pessoas com transtornos do espectro do autismo (pp. 56-71). São Paulo: Memnon Edições Científicas.

Ferguson, J., Craig, E. A., \& Dounavi, K. (2019). Telehealth as a model for providing behaviour analytic interventions to individuals with autism spectrum disorder: A systematic review. Journal of autism and developmental disorders, 49(2), 582-616. doi: 10.1007/s10803-018-3724-5

Ferster, C. B. (1967). Arbitrary and natural reinforcement. Psychological Record, 17, 341-347.
Flannery, K. B., \& Horner, R. H. (1994). The relationship between predictability and problem behavior for students with severe disabilities. Journal of Behavioral Education, 4, 157-176. doi: 10.1007/BF01544110

Fialho, J. P. G. (2018). Uso de pistas visuais. Em C. P. Duarte, L. C. Silva, \& R. L. Velloso (Orgs.), Estratégias da análise do comportamento aplicada para pessoas com transtorno do espectro do autismo (pp. 195-217). São Paulo: Memnon Edições Científicas.

Graziano, A. M. (1969). Clinical innovation and the mental health power structure: A social case history. American Psychologist, 24(1), 10-18. doi: $10.1037 / \mathrm{h} 0027209$

Grupo MAVE (2020, 02 abril). Estimativa de risco de espalhamento da COVID-19 nos estados brasileiros $e$ avaliação da vulnerabilidade socioeconômica nos municípios. Relatório n. 3. Recuperado de: http://covid-19.procc.fiocruz.br/

Hall, G., \& Sundberg, M. L. (1987). Teaching mands by manipulating conditioned establishing operations. The Analysis of verbal behavior, 5, 41-53. doi: 10.1007/BF03392819

Hume, K., \& Smith, S. (2009). Steps for implementation: Visual supports. Chapel Hill, NC: The National Professional Development Center on Autism Spectrum Disorders, Frank Porter Graham Child Development Institute, The University of North Carolina.

Johnson, B. M., Miltenberger, R. G., Egemo-Helm, K., Jostad, C. M., Flessner, C., \& Gatheridge, B. (2005). Evaluation of behavioral skills training for teaching abduction-prevention skills to young children. Journal of applied behavior analysis, 38(1), 67-78. 10.1901/jaba.2005.26-04

Johnson, C. R., Handen, B. L., Butter, E., Wagner, A., Mulick, J., Sukhodolsky, D. G., ... \& Aman, M. G. (2007). Development of a parent training program for children with pervasive developmental disorders. Behavioral Interventions, 22, 201-221. doi: 10.1002/bin.237

Longano, J. M., \& Greer, R. D. (2006). The effects of a stimulus-stimulus pairing procedure on the acquisition of conditioned reinforcement on observing and manipulating stimuli by young children with autism. Journal of Early and Intensive Behavior Intervention, 3(1), 62-80. doi: 10.1037/h0100323

Massey, N. G., \& Wheeler, J. J. (2000). Acquisition and generalization of activity schedules and their effects on task engagement in a young child with autism in an inclusive pre-school classroom. Education and Training in Mental Retardation and Developmental Disabilities, 35(3), 326-335.

Michael, J. (1982). Distinguishing between discriminative and motivational functions of stimuli. Journal of Experimental Analysis of Behavior, 37, 149-155. doi: 10.1901\%2Fjeab.1982.37-149

Miguel, C. F. (2000). O conceito de operação estabelecedora na análise do comportamento. Psicologia: Teoria e Pesquisa, 16(3), 259-267. doi: 10.1590/S0102-37722000000300009 
Morrison, R. S., Sainato, D. M., Benchaaban, D., \& Endo, S. (2002). Increasing play skills of children with autism using activity schedules and correspondence training. Journal of early intervention, 25(1), 58-72. doi: $10.1177 \% 2$ F105381510202500106

National Autism Center (2015). Findings and conclusions: National standards project, phase 2. Randolph (MA): National Autism Center.

Peterson, K. M., Piazza, C. C., Luczynski, K. C., \& Fisher, W. W. (2017). Virtual-care delivery of appliedbehavior-analysis services to children with autism spectrum disorder and related conditions. Behavior Analysis: Research and Practice, 17(4), 286-297. doi: 10.1037/bar0000030

Roane, H. S., Vollmer, T. R., Ringdahl, J. E., \& Marcus, B. A. (1998). Evaluation of a brief stimulus preference assessment. Journal of Applied Behavior Analysis, 31, 605-620. doi: 10.1901\%2Fjaba.1998.31-605

Comunidad Los Horcones. (1992). Natural reinforcement: a way to improve education. Journal of Applied Behavior Analysis, 25, 71-75. doi: 10.1901\%2Fjaba.1992.25-71

Schreibman, L., Dawson, G., Stahmer, A. C., Landa, R., Rogers, S. J., McGee, G. G., Kasari, C., Ingersoll, B., Kaiser, A. P., Bruinsma, Y., McNerney, E., Wetherby, A., \& Halladay, A. (2015). Naturalistic Developmental Behavioral Interventions: Empirically Validated Treatments for Autism Spectrum Disorder. Journal of autism and developmental disorders, 45(8), 24112428. doi: 10.1007/s10803-015-2407-8

Sella, A. C., \& Ribeiro, D. M. (Eds.). (2018). Análise do comportamento aplicada ao transtorno do espectro autista. Curitiba: Appris.

Silva, A. J. M., Barboza, A. A., Miguel, C. F. A., \& Barros, R. S. (2019). Evaluating the efficacy of a parent-implemented autism intervention program in Northern Brazil. Temas em Psicologia, 27, 523-532. doi: 10.9788/tp2019.2-16

Smith, S., \& Collet-Klingenberg, L. (2009). Implementation checklist for visual boundaries. Madison, WI: The National Professional Development Center on Autism Spectrum Disorders, Waisman Center, University of Wisconsin.

Sterling-Turner, H. E., \& Jordan, S. S. (2007). Interventions addressing transition difficulties for individuals with autism. Psychology in the Schools, 44(7), 681-690. doi: 10.1002/pits.20257

Vismara, L. A., McCormick, C., Young, G. S., Nadhan, A., \& Monlux, K. (2013). Preliminary findings of a telehealth approach to parent training in autism. Journal of Autism and Developmental Disorders, 43(12), 2953-2969. doi: https://doi.org/10.1007/s10803-013-1841-8

Yamamoto, M. E., \& Carvalho, A. M. A. (2002). Brincar para quê? Uma abordagem etológica ao estudo da brincadeira. Estudos de Psicologia, 7(10), 163-164. doi: 10.1590/S1413-294X2002000100016

Windholz, M. H. (2016). Passo a passo, seu caminho: guia curricular para o ensino de habilidades básicas. São Paulo: EDICON.
Wong, C., Odom, S. L., Hume, K. A., Cox, A. W., Fettig, A., Kucharczyk, S., ... \& Schultz, T. R. (2015). Evidence-based practices for children, youth, and young adults with autism spectrum disorder: A comprehensive review. Journal of Autism and Developmental Disorders, 45(7), 1951-1966. doi: 10.1007/s10803-014-2351-z

Submetido em: 29/04/2020 Aceito em: 08/05/2020 